\title{
Theoretical study of the surface energy and surface relaxation of the $W(001)$ surface
}

\author{
C. T. Chan and Steven G. Louie \\ Department of Physics, University of California, Berkeley, California 94720 \\ and Materials and Molecular Research Division, Lawrence Berkeley Laboratory, \\ Berkeley, California 94720 \\ (Received 21 October 1985)
}

\begin{abstract}
The structural properties of the $\mathrm{W}(001)(1 \times 1)$ surface are calculated using a fully self-consistent pseudopotential linear combination of atomic obitals method. The surface energy [3.09 eV/(surface atom)], the top-layer relaxation $(5.9 \%)$, and the relaxation energy $(0.059 \mathrm{eV})$ we obtained are in good agreement with available experimental data and recent all-electron full-potential linear augmented-plane-wave results. The results demonstrate that the method can treat the structural properties of transition-metal surfaces accurately.
\end{abstract}

The W(001) surface has long been the "standard" transition-metal surface for experimental surface-science studies. There are rich and interesting surface phenomena including the existence of surface states, surface relaxation, clean and absorbate-induced surface reconstruction. ${ }^{1}$ Lowenergy electron diffraction (LEED) ${ }^{2}$ and ion backscatteringchanneling $^{3}$ experiments suggest that the top layer of $\mathrm{W}(001)$ contracts by about $6 \%$. However, the relaxation, as determined by comparing experimental and calculated LEED $I-V$ profiles, can vary substantially from one experiment to the other. ${ }^{2}$ Surface energies are even more difficult to measure accurately. Accurate first-principles calculations are thus highly desirable. In the present study, we found that the top layer has an equilibrium contraction of $5.9 \%$, in good agreement with experiments. The surface energy is $\sim 3.1 \mathrm{eV} /$ (surface atom) and the relaxation energy is 0.059 $\mathrm{eV} /$ (surface atom).

Although the surface structural properties of semiconductors have been studied for several years using total-energy methods, ${ }^{4}$ it was not until very recently that total-energy calculations for transition-metal surfaces could be performed accurately from first principles. $^{5-9}$ Most of the previous studies of transition-metal surface structural properties were treated by all-electron methods such as the full-potential linear augmented-plane-wave (FLAPW) ${ }^{10}$ and self-consistent local orbital (SCLO) ${ }^{11}$ schemes. In this work, the surface energy and top-layer relaxation of W(001) are calculated using a pseudopotential linear combination of atomic orbitals (LCAO) method. ${ }^{12}$ This method has been applied to insulators and semiconductors. ${ }^{13}$ Recently, it was extended to include full self-consistency ${ }^{14}$ and has treated successfully the electronic and structural properties of bulk transition-metal systems. Our results for the W(001) surface agree well with the recent all-electron FLAPW results of Fu et al. ${ }^{6,7}$

In the present work, the electron-electron interactions are treated by the density-functional formalism within the local-density approximation (LDA), ${ }^{15}$ using the Wigner exchange-correlation potential ${ }^{16}$ in the Kohn-Sham equations. Scalar relativistic ionic pseudopotentials ${ }^{17}$ are used for the $\mathrm{W}$ ions. The nonlinearity of the exchange-correlation interaction between valence and core electrons is treated by the partial-core method. ${ }^{18}$ The pseudo-wave-functions are expanded in an LCAO basis:

$$
\phi_{i}(\mathbf{k}, \mathbf{r})=\frac{1}{\sqrt{\Omega}} \sum_{\mathbf{R}} e^{i \mathbf{k} \cdot\left(\mathbf{R}+\tau_{i}\right)} f_{i}\left(\mathbf{r}-\mathbf{R}-\tau_{i}\right),
$$

where $\Omega$ is the crystal volume, $\mathbf{R}$ is a lattice vector, $\boldsymbol{\tau}_{i}$ is a basis vector, and $f(\mathbf{r})$ are localized functions of the form

$$
f_{\alpha / m}(\mathbf{r})=A_{\alpha l m} e^{-\alpha r^{2}} r^{l} K_{l m}(\theta, \phi),
$$

where $A_{\alpha l m}$ are normalization constants and $K_{l m}$ are Kubic harmonics.

The calculation is done fully self-consistently by a scheme which has been presented in detail elsewhere. ${ }^{14}$ Basically, we compute and store the Fourier components of the overlap matrices of the basis orbitals for every $\mathbf{k}$ point:

$$
\pi_{i j}(\mathbf{k}, \mathbf{G})=\left\langle\mathbf{k}, j\left|\frac{e^{-i \mathbf{G} \cdot \mathbf{r}}}{\Omega_{c}}\right| \mathbf{k}, i\right\rangle
$$

where $\Omega_{c}$ is the volume of the unit cell and $|\mathbf{k}, i\rangle$ denote basis orbitals. The charge density and the change in the Hamiltonian matrix elements in every iteration can then be calculated straightforwardly as

$$
\rho(\mathbf{G})=\sum_{n, \mathbf{k}} \psi_{n \mathbf{k}}^{\dagger} \underline{\pi}(\mathbf{k}, \mathbf{G}) \psi_{n \mathbf{k}},
$$

and

$$
\delta H_{i j}(\mathbf{k})=\sum_{\mathbf{G}} \delta V(\mathbf{G}) \pi_{i j}^{*}(\mathbf{k}, \mathbf{G}) \Omega_{c},
$$

where $\psi_{n k}$ here is the eigenvector in the basis space corresponding to a wave function and $\delta V$ is the change in the screening potentials. We can achieve full (point-to-point) self-consistency efficiently with no shape constraint or approximation to the crystal potential. The self-consistency criterion was that the Fourier components of the difference between the input and output potentials were to have a maximum of less than $1 \mathrm{mRy}$. As the total energy is carefully formulated as an explicit functional of the output charge density only, ${ }^{12}$ the total energy was determined stable to at least $10^{-4} \mathrm{eV}$.

The W(001) surface is modeled by slabs of 3,5 , and 7 layers of $\mathrm{W}$ atoms. As the charge and total energy are evaluated in Fourier space, it is more convenient for us to use the "supercell geometry," i.e., the W slabs are repeated in the (001) direction. Adjacent slabs are separated by a vacuum equivalent to 8 layers of $W$. This thickness prevents adjacent slabs from interacting with one another. We have used three decays $(\alpha=4.0,0.89$, and 0.2$)$ for the radial part of the localized orbitals and angular harmonics up to $l=2$ (i.e., 30 orbitals for each $W$ atom). When applied to bulk $W$ in the bcc structure, this set of orbitals gives 
TABLE I. Structural and cohesive properties of bulk bcc W using three radial decays $(\alpha=0.2,0.89$, and 4.0$)$ and four decays $(\alpha=0.2$ $0.49,1.22$, and 3.0). Experimental values are quoted from C. Kittel, Introduction to Solid State Physics (Wiley, New York, 1976).

\begin{tabular}{|c|c|c|c|}
\hline & $\begin{array}{l}\text { Three } \\
\text { decays }\end{array}$ & $\begin{array}{c}\text { Four } \\
\text { decays }\end{array}$ & Experiment \\
\hline Lattice constant $(\AA)$ & 3.10 & 3.13 & 3.16 \\
\hline Bulk modulus (Mbar) & 3.69 & 3.34 & 3.23 \\
\hline Cohesive energy $(\mathrm{eV})$ & 7.93 & 8.46 & 8.90 \\
\hline
\end{tabular}

equilibrium structural properties as summarized in Table $\mathrm{I}$. The lattice constant is about $1.8 \%$ smaller than the experimental value. In the surface calculations, the unrelaxed surface is taken to have the lattice parameter corresponding to the calculated equilibrium value of bulk bcc $W$ of the present basis set. We can achieve an even more accurate description of bulk structural properties (e.g., lattice parameter within $1 \%$ of experiment) if four instead of three decays are used in the radial wave functions (Table I). However, we have chosen to use the three-radial-decay basis set for the present work to keep the Hamiltonian matrix smaller. The error introduced should be very small as long as we start relaxing the surface from its bulk equilibrium positions as determined by the basis chosen.

In the surface-energy calculations, atomic orbitals are put in the vacuum region one layer above the surface $W$ atoms (on both sides of the slab) at positions where there would be $\mathbf{W}$ atoms if it were bulk bcc $\mathbf{W}$. This is to ensure that the basis set is equivalently complete for the surface atoms since there is no $\mathbf{W}$ atom in the vacuum region. Three $\mathbf{k}$ points in the irreducible surface Brillouin zone are used. The total pseudoenergy of the slab, $E_{n}$ ( $n$ denotes $n$ layers of $\mathbf{W}$ ), is calculated self-consistently and the surface energy per surface atom is determined by

$$
E_{s}=\frac{1}{2}\left(E_{n}-n E_{b}\right) \text {, }
$$

where $E_{b}$ is the total energy of a $\mathrm{W}$ atom in a bulk environment. $E_{b}$ is determined by calculating the total energy of bulk bcc W with a unit cell corresponding to six layers of $\mathrm{W}$ in the (001) direction with the same basis set and $\mathbf{k}$ points. We obtained $E_{b}=-274.341 \mathrm{eV}$ and, with $E_{7}=-1914.205$ $\mathrm{eV}, E_{s}$ is calculated to be $3.09 \mathrm{eV} /$ (surface atom).

To check the precision of the above procedure, we also calculated the total energy of five- and three-layer slabs (with same basis set, vacuum thickness, and $\mathbf{k}$ points). Results are shown in Table II. The total energy of a slab of $n$ layers of $\mathrm{W}$ atoms can be written as

$$
E_{n}=n E_{b}+2 E_{s} \text {, }
$$

from which we can determine $E_{s}$ and $E_{b}$ simultaneously if

TABLE II. Total energy $E_{n}$ (in the pseudopotential formalism) of a W(001) slab of $n$ layers of thickness.

\begin{tabular}{cccc}
\hline \hline$n$ & 3 & 5 & 7 \\
\hline$E_{n}(\mathrm{eV})$ & -816.637 & -1365.532 & -1914.205 \\
\hline
\end{tabular}

we have $E_{n}$ for two or more values of $n$. This method has been used by Richter, Smith, and $\mathrm{Gay}^{5}$ and has no explicit reference to bulk calculations. Using $n=7$ and 5 in Eq. (7), we obtained $E_{b}=-274.336 \mathrm{eV}$ and $E_{s}=3.07 \mathrm{eV}$, in essentially perfect agreement with the values obtained by subtracting bulk and surface energies. If we use $n=3,5$, and 7, and a least-squares fit to Eq. (7), we find $E_{s}=3.25 \mathrm{eV}$. This slightly higher $E_{s}$ is due to the fact that a three-layer slab is too thin and has higher surface energy because even the atoms in the middle layer have a lot of neighbors missing. Moreover, using $E_{n}$ for $n=5$ in Eq. (6) gives the same $E_{\mathrm{s}}=3.09 \mathrm{eV}$ as for $n=7$. This gives us confidence that seven layers of $\mathrm{W}$ is enough for the surface-energy determination. It also indicates that the interaction between the W layers is short ranged.

The calculated surface energy agrees very well with the recent FLAPW results ${ }^{6}$ (see Table III) and is substantially higher than those obtained by empirical tight-binding (TB) methods. ${ }^{19,20}$ Comparing the calculated surface energy ( $T=0 \mathrm{~K}$ ) with experiment is difficult since the surface free energies are measured at finite temperature with large variation depending on the method of determination. For example, at $\sim 2000 \mathrm{~K}$, the surface free energy of $\mathrm{W}$ was found to be $1810 \pm 630 \mathrm{ergs} / \mathrm{cm}^{2}$ by multiphase equilibration techniques $^{21}$ and $2800 \pm 280 \mathrm{ergs} / \mathrm{cm}^{2}$ by pulsed field-emission techniques. ${ }^{22}$ The surface energy has been measured at 77 and $4 \mathrm{~K}$ by the crack propagation method ${ }^{23}$ (with strong dependence on experimental conditions) to be $\sim 6000$ $\mathrm{ergs} / \mathrm{cm}^{2}$, which is about $3.7 \mathrm{eV} /$ (surface atom) if the surface is assumed to be (001).

In the present study, the surface relaxation is determined by relaxing the surface layer, keeping the position of all atoms in the interior fixed. The results are plotted in Fig. 1. The ideal surface is taken to be the reference and the relaxation energy is defined as the energy gained with respect to the ideal surface by relaxation. A polynomial with nonzero second- and third-order coefficients (solid line in Fig. 1) fitted to the calculated points gives an equilibrium relaxation of $5.9 \%$ and a relaxation energy of $0.059 \mathrm{eV}$. From the harmonic coefficient, the frequency of vibration of the surface layer is found to be $-4.5 \mathrm{THz}$, in good agreement with the $4.3 \mathrm{THz}$ found by Fu et al. ${ }^{7}$ To check the sensitivity of the calculated relaxation on the convergence of the basis, a calculation was performed with the extra orbitals in the vacuum taken away (i.e., using only on-site Gaussians). The

TABLE III. Comparison of theoretical results on the structural properties of $\mathrm{W}(001)$. The surface energy and relaxation energy are in $\mathrm{eV} /$ (surface atom). The $\%$ relaxation and relaxation energy are

\begin{tabular}{|c|c|c|c|c|}
\hline & \multicolumn{2}{|c|}{$\begin{array}{c}\text { Fully } \\
\text { self-consistent }\end{array}$} & \multicolumn{2}{|c|}{$\begin{array}{c}\text { Empirical } \\
\text { tight-binding }\end{array}$} \\
\hline & $\mathrm{LCAO}^{\mathrm{a}}$ & FLAPW $^{\mathrm{b}}$ & $\mathrm{TB} 1^{\mathrm{c}}$ & TB2 $^{\mathrm{d}}$ \\
\hline Surface energy $(\mathrm{eV})$ & 3.09 & 3.18 & $\cdots$ & 1.9 \\
\hline$\%$ relaxation & 5.9 & 5.5 & 4.0 & 3.0 \\
\hline Relaxation energy $(\mathrm{eV})$ & 0.059 & 0.048 & 0.027 & .. \\
\hline
\end{tabular}
for the top layer.

${ }^{\text {aPresent }}$ calculation.

${ }^{\text {b}}$ Fu et al. (Refs. 6 and 7)

'Terakura, Terakura, and Hamada (Ref. 20).

${ }^{\mathrm{d}}$ Treglia, Desjonquere, and Spanjaard (Ref. 19). 


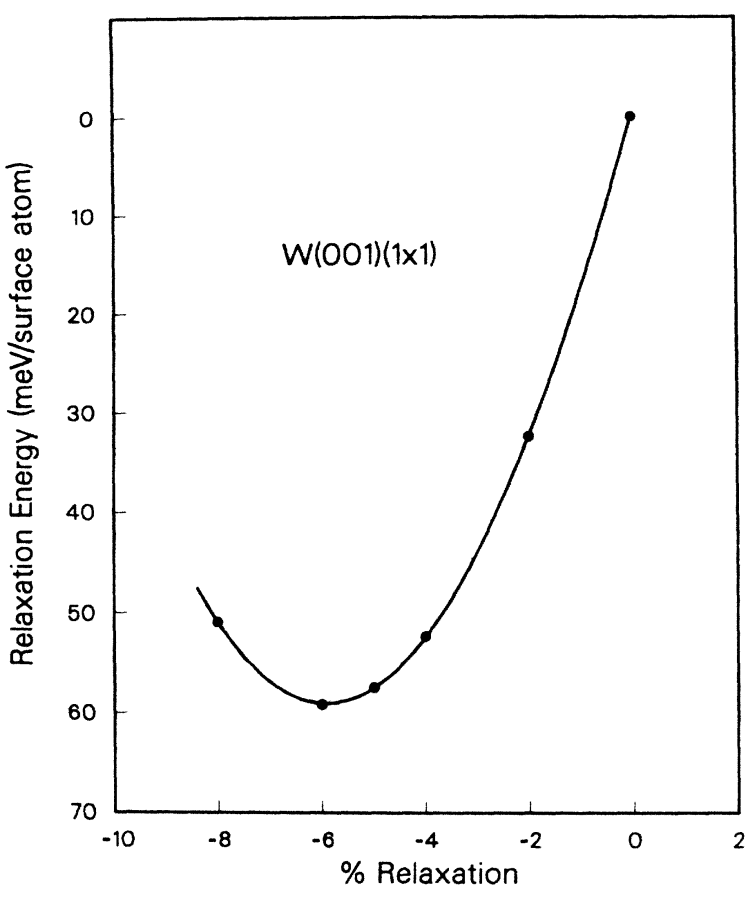

FIG. 1. Relaxation energy of W(001) surface as a function of the relaxation of the top layer. Relaxation is expressed in terms of the percentage change of the interlayer spacing. Negative numbers indicate inward relaxation.

surface energy becomes $4.0 \mathrm{eV}$, indicating that the surface energy is sensitive to the completeness of basis near the vacuum region. However, the equilibrium relaxation position of the surface layer, relaxation energy, and the surface vibration frequency are found to be $5.6 \%, 0.054 \mathrm{eV}$, and 4.6 $\mathrm{THz}$, respectively, in very close agreement with those of the more complete basis set, i.e., with orbitals in the vacuum. When the number of $\mathbf{k}$ points is doubled, the surface energy (calculated with on-site Gaussians only) changes by less than $5 \%$. This small change and the fact that our results agree with experimental data and previous self-consistent calculations $s^{6,7}$ indicate that the surface energy and relaxation for $\mathbf{W}(001)$ can be calculated with a relatively small $\mathbf{k}$-point set.

The calculated surface energy of $W(001) \quad[\sim 3.1$ $\mathrm{eV} /$ (surface atom)] is rather high because $\mathrm{W}$ has the highest cohesive energy of $8.9 \mathrm{eV}$ in the periodic table. It is interesting to note that the ratio of the theoretical surface energy to the cohesive energy $(3.1 / 7.9)$ is very close to the ratio of the number of missing nearest neighbors to the total number of nearest neighbors $(5 / 14)$, counting both first and second neighbors for the bcc structure. This is consistent with a simple picture that the surface energy is just the energy needed to break nearest-neighbor bonds when the (001) surface is formed. The top layer relaxes inwards so that $\mathrm{W}$ atoms on the surface form stronger $d$ bonds with atoms in the interior. Equilibrium is reached when the increase in the kinetic energy of the electrons (which become more confined when the top interlayer distance decreases) counterbalances the gain in the potential energy. The relaxation energy $(59 \mathrm{meV})$ is less than $2 \%$ of the total surface energy.

The present results are summarized in Table III. They are compared with first-principles self-consistent ${ }^{6,7}$ and empirical tight-binding results. ${ }^{19,20}$ We may conclude that, at least for the case of $W(001)$, the agreement between firstprinciples local-density approximation calculations is better than the agreement among existing LEED data. ${ }^{2}$ This establishes the credibility of the theoretical schemes (pseudopotential LCAO and FLAPW). It demonstrates the versatility of the pseudopotential LCAO method in surface calculations: It can treat transition-metal surfaces as well as insulator surfaces ${ }^{13}$ in calculations of structural properties.

We would like to thank D. Vanderbilt, J. R. Chelikowsky, M. Hybertsen, and S. Fahy for helpful discussions and suggestions. This work was supported by the Director, Office of Energy Research, Office of Basic Sciences, Materials Sciences Division of the U.S. Department of Energy under Contract No. DE-AC03-76SF00098. CRAY computer time at the National Magnetic Fusion Energy Computer Center was provided by the U.S. Office of Energy Research of the Department of Energy.
${ }^{1}$ For a review, see, D. A. King, Phys. Scr. T4, 34 (1983), and references therein.

${ }^{2}$ M. A. Van Hove and S. Y. Tong, Surf. Sci. 54, 91 (1976); B. W. Lee, A. Ignatiev, S. Y. Tong, and M. A. Van Hove, J. Vac. Sci. Technol. 14, 291 (1977); M. K. Debe, D. A. King, and F. S. March, Surf. Sci. 68, 437 (1977); L. J. Clarke and L. M. Garza, ibid. 99, 419 (1980); R. Feder and J. Kirschner, ibid. 103, 75 (1981).

${ }^{3}$ L. C. Feldman, R. L. Kauffmann, P. J. Silverman, R. A. Zuhr, and J. H. Barrett, Phys. Rev. Lett. 39, 38 (1977).

${ }^{4}$ For a review, see, M. L. Cohen and S. G. Louie, Annu. Rev. Phys. Chem. 35, 537 (1984).

${ }^{5}$ R. Richter, J. R. Smith, and J. G. Gay, in The Structure of Surfaces, edited by M. A. Van Hove and S. Y. Tong, Springer Series in Surface Sciences, Vol. 2 (Springer-Verlag, Berlin, 1985), p. 35

${ }^{6}$ C. L. Fu, S. Ohnishi, H. J. F. Jansen, and A. J. Freeman, Phys. Rev. B 31, 1168 (1985).

${ }^{7}$ C. L. Fu, S. Ohnishi, E. Wimmer, and A. J. Freeman, Phys. Rev. Lett. 53, 675 (1984)

${ }^{8}$ C. L. Fu, A. J. Freeman, E. Wimmer, and M. Weinert, Phys. Rev.
Lett. 54, 2261 (1985)

${ }^{9} \mathrm{C}$. Umrigar and J. W. Wilkins, Phys. Rev. Lett. 54, 1551 (1985).

${ }^{10}$ E. Wimmer, H. Krakauer, M. Weinert, and A. J. Freeman, Phys. Rev. B 24, 864 (1981).

11J. R. Smith, J. G. Gay, and F. J. Arlinghaus, Phys. Rev. B 21, $2201(1980)$

12J. R. Chelikowsky and S. G. Louie, Phys. Rev. B 29, 3470 (1984); J. R. Chelikowsky, S. G. Louie, D. Vanderbilt, and C. T. Chan, Int. J. Quantum Chem. Quantum Chem. Symp. 18, 105 (1984).

${ }^{13}$ D. Vanderbilt and S. G. Louie, Phys. Rev. B 29, 7099 (1984); 30, 6118 (1984); D. Vanderbilt, S. G. Louie, and M. L. Cohen, Phys. Rev. Lett. 53, 1477 (1984); M. Hanfland, K. Syassen, S. Fahy, S. G. Louie, and M. L. Cohen, Phys. Rev. B 31, 6896 (1985); S. L. Richardson, M. L. Cohen, S. G. Louie, and J. R. Chelikowsky, Phys. Rev. Lett. 54, 2549 (1985).

${ }^{14}$ C. T. Chan, D. Vanderbilt, and S. G. Louie, Phys. Rev. B (to be published)

15P. Hohenberg and W. Kohn, Phys. Rev. 136, B864 (1964); W. Kohn and L. J. Sham, ibid. 140, A1133 (1965); in Theory of the Inhomogeneous Electron Gas, edited by N. H. March and 
S. Lundqvist (Plenum, New York, 1983).

${ }^{16}$ E. Wigner, Phys. Rev. 46, 1002 (1934).

${ }^{17}$ L. Kleinman, Phys. Rev. B 21, 2630 (1980); G. B. Bachelet and M. Schluter, ibid. 25, 2103 (1982).

${ }^{18}$ S. G. Louie, S. Froyen, and M. L. Cohen, Phys. Rev. B 26, 1738 (1982).

${ }^{19}$ G. Treglia, M. C. Desjonquere, and D. Spanjaard, J. Phys. C 16,
2407 (1983).

${ }^{20}$ I. Terakura, K. Terakura, and N. Hamada, Surf. Sci. 103, 103 (1981).

${ }^{21}$ B. C. Allen, J. Less-Common Met. 29, 263 (1972).

22P. C. Bettler and G. Barnes, Surf. Sci. 10, 165 (1968).

${ }^{23}$ J. E. Cordwell and D. Hull, Philos. Mag. 19, 951 (1969). 